\title{
Assessment of selected parameters of the circulatory system in patients undergoing oral aspirin challenge
}

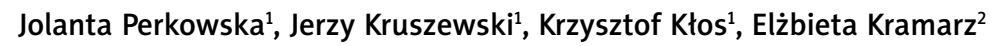 \\ ${ }^{1}$ Department of Infectious Diseases and Allergology, Military Institute of Medicine, Warsaw, Poland \\ ${ }^{2}$ Department of Cardiology and Internal Diseases, Military Institute of Medicine, Warsaw, Poland
}

Adv Dermatol Allergol 2020; XXXVII (4): 579-583

DOI: https://doi.org/10.5114/ada.2019.88623

\begin{abstract}
Introduction: It is known that the administration of the drug during the oral aspirin challenge (OAC) can cause hypersensitivity symptoms not only from the respiratory system or skin, but also from the cardiovascular system. Aim: To assess the occurrence and nature of cardiovascular adverse events during the OAC in patients suspected of hypersensitivity to nonsteroidal anti-inflammatory drugs (NSAIDs).

Material and methods: The study included 52 patients with symptoms of hypersensitivity to aspirin (ASA) or other NSAIDs in the form of skin reactions or respiratory response in anamnesis. Patients were treated with OAC and simultaneously were subject to monitoring of clinical manifestations of hypersensitivity to ASA/NSAIDs, ventilation disorders and cardiovascular functions.

Results: The most common reaction of the cardiovascular system during OAC was tachycardia or supraventricular and ventricular extrasystoles, regardless of the day of the study and the result of OAC. Supraventricular and ventricular tachycardia was recorded incidentally. Atrial or ventricular fibrillation or flutter was not observed. There was no evidence of any ischemic heart disease. In 2 patients, hypotension was registered, but only 1 of them required typical treatment of anaphylaxis.

Conclusions: No clinically significant cardiac arrhythmias were recorded during OAC. The changes observed in the records of blood pressure and ECG monitoring show that OAC performed in accordance with the current guidelines does not pose a high risk to the patient's health and life as a result of cardiovascular reactions.
\end{abstract}

Key words: aspirin, hypersensitivity, oral aspirin challenge, Kounis syndrome, arrhythmia.

\section{Introduction}

The discovery of acetylsalicylic acid in 1897 opened a new era for the use of non-steroids anti-inflammatory drugs (NSAIDs) [1]. Already a few years after the discovery of the drug, the first cases of hypersensitivity were described $[2,3]$. The widespread use of NSAIDs increases the incidence of hypersensitivity to the drug, which in the general population may reach $0.3-0.5 \%$ [4]. In the adult population with diagnosed bronchial asthma hypersensitivity to aspirin (ASA) it may even amount to $21 \%[4,5]$. The enormous scale of the problem gives rise to verification of the symptoms reported by patients. For this purpose drug provocation tests are used, and in this work oral aspirin challenge (OAC) was used.

The OAC is considered the gold standard in the diagnosis of hypersensitivity to NSAIDs [6]. It has the highest sensitivity and specificity, but due to higher cumulative doses, high exposure to the drug of all effector organs (such as the respiratory system, skin, mucous membranes, digestive system or circulatory system) and no possibility of disruption (long absorption time by the gastrointestinal tract), it may have many adverse effects [7]. During the test, special attention is paid to the clinical symptoms of hypersensitivity especially from the skin and respiratory system. Objective monitoring of ventilation disorders with the use of a spirometer was carried out. On the other hand, circulatory functions are not routinely monitored, apart from blood pressure measurements in the search for hypotension as a result of severe anaphylaxis. Meanwhile, the available literature on the subject presents works describing various reactions of the circulatory system during anaphylaxis, e.g. myocardial infarction as a result of the coronary artery contraction or destabilization of the atherosclerotic plaque, or stent thrombosis under the influence

Address for correspondence: Jolanta Perkowska MD, PhD, Department of Infectious Diseases and Allergology, Military Institute of Medicine, 128 Szaserów St, 04-141 Warsaw, Poland, phone: +48 261817 519, e-mail: jperkowska@wim.mil.pl Received: 13.08 .2019 , accepted: 6.09.2019. 
of mediators of the allergic reaction - called Kounis syndrome $[8,9]$, described in patients with hypersensitivity to hymenoptera venom, hypersensitivity to drugs, including aspirin [10] and ibuprofen [11].

It is also known that histamine, through the receptors in the heart, also affects the cardiac-conduction system $[12,13]$. The literature describes studies in which heart disorders were recorded such as atrial fibrillation or ventricular tachycardia during anaphylaxis [14, 15].

\section{Aim}

The aim of the study was to assess the incidence and nature of cardiovascular side effects in patients suspected of being hypersensitive to ASA/NSAIDs and subjected to OAC, their relation with the outcome of the challenge and the safety evaluation of the test.

\section{Material and methods}

The study included 52 patients hospitalized in the clinic in 2012-2016 with signs of hypersensitivity to ASA and/or NSAIDs in the form of urticaria and/or angioedema $(n=47)$, bronchospasm $(n=11)$, including

Table 1. Test protocol

\begin{tabular}{c}
\hline Oral aspirin challenge \\
\hline Day 1 - placebo \\
\hline Monitoring of clinical symptoms of hypersensitivity \\
\hline Monitoring of ventilation disorders - FEV \\
\hline BP and ECG monitoring
\end{tabular}

Table 2. Oral aspirin challenge diagram

\begin{tabular}{lcc}
\hline Day 1 - placebo & \multicolumn{2}{c}{ Day 2 - ASA } \\
\hline Doses & Dose [mg] & $\begin{array}{c}\text { Cumulative dose } \\
{[\mathrm{mg}]}\end{array}$ \\
\hline 1. Sacchari lactis & 27 & 27 \\
\hline 2. Sacchari lactis & 44 & 71 \\
\hline 3. Sacchari lactis & 117 & 188 \\
\hline 4. Sacchari lactis & 312 & 500 \\
\hline 5. Sacchari lactis & 500 & 1000 \\
\hline
\end{tabular}

the mixed form $(n=6)$ in the history, with normal ECG and spirometry. The OAC was performed a minimum of 7 days after discontinuation of antihistamines, while using a reliever-free diet (without histamine) in a symptoms-free period. The patients qualified for the study were subjected to a 2-day single-blind challenge test with ASA, with simultaneous monitoring of clinical manifestations of ASA hypersensitivity, ventilation and cardiovascular function disorders according to the protocol in Table 1.

Particular attention was paid to skin and mucous membrane symptoms such as urticaria, angioedema, erythema, pruritus and the respiratory system: dyspnoea, cough, rhinitis, bronchospasm and a decrease in forced expiratory volume in $1 \mathrm{~s}$ (FEV) (AbcMED's PNEUO $\mathrm{NEB}^{\mathrm{TM}}$ spirometer). During the entire study (2 days), the cardiovascular system was monitored using 24-hour registration of ECG recording (Holter ECG) with a Reynolds Medical device (US) as well as daily blood pressure monitoring (DBPM) using a BOSO device (Bosch \& Sohn, Germany). The provocation test was carried out according to the protocol by Professor Niżankowska (Table 2) [16].

On the first day, placebo (lactose) was administered orally 5 times every $1.5-2.0 \mathrm{~h}$ in identical-looking capsules; on the second day, doses of ASA were increased up to a total dose of $500 \mathrm{mg}$ or in the absence of any reaction - up to a maximum dose of $1000 \mathrm{mg}$ according to Table 2 [16]. The test was discontinued at the time of occurrence of clinical manifestations of hypersensitivity and/or a decrease in $\mathrm{FEV}_{1} \geq 20 \%$ of the output value and/ or the appearance of hypotension (Table 3 ). The study was approved by the Military Institute of Medicine Ethics committee.

\section{Statistical analysis}

In terms of descriptive statistics, percentages, arithmetic averages and standard deviations were calculated. In order to find the significance of differences in cardinality in the groups, the $\chi^{2}$ test was applied, while the comparison of some parameters in individual groups was carried out using the Student's $t$ test for independent variables, assuming significant differences in $p$-values at $<0.05$. Calculations were performed using the Statistica software (StatSoft Poland Ltd. 2015, medical kit version 3.0).

Table 3. Criteria for the recognition of oral aspirin challenge as positive

\begin{tabular}{|c|c|c|c|}
\hline Day 1 - placebo & & Day 2 - ASA & \\
\hline $\begin{array}{l}\text { Clinical symptoms of } \\
\text { hypersensitivity } \\
\downarrow \mathrm{FEV}_{1} \geq 15 \% \text { of the output } \\
\text { value } \\
\text { Hypotension }\end{array}$ & $\begin{array}{c}\text { Clinical symptoms of } \\
\text { hypersensitivity: urticaria, } \\
\text { swelling, runny nose, dyspnoea, } \\
\text { exacerbation of bronchial } \\
\text { asthma, abdominal pain, } \\
\text { diarrhoea }\end{array}$ & $\begin{array}{c}\downarrow \mathrm{FEV}_{1} \geq 20 \% \text { of the } \\
\text { output value }\end{array}$ & $\begin{array}{c}\text { Hypotension registered by } \\
\text { DBPM (along with clinical } \\
\text { manifestations } \\
\text { of hypersensitivity) }\end{array}$ \\
\hline Disqualification & & sitive result of the te & \\
\hline
\end{tabular}




\section{Results}

A positive OAC result was obtained in $42 \%$ of patients. The most frequently observed clinical manifestation of OAC (+) was cutaneous urticarial-oedema lesions of various severity $-78 \%$ ( $n=17)$, including an isolated form $\sim 60 \%(n=13)$, whereas $18 \%(n=4)$ simultaneously manifested urticaria/angioedema and a feeling of chest discomfort or dyspnoea. The isolated obstruction was manifested by $23 \%$ of patients $(n=5)$ (Figure 1$)$.

The most common reaction of the cardiovascular system during OAC was tachycardia ( $80 \%$ of patients), regardless of the day of the study and the result of OAC. Similarly, supraventricular extrasystoles (SVEx) and ventricular extrasystoles (Vex) occurred with a comparable frequency on both days, regardless of the test result. Both SVT and ventricular tachycardia (VT) were registered in several patients, whereas the disorders occurred both on the day of placebo and ASA administration. In 1 patient, SVT occurred only on the day of ASA administration. The patients did not feel any discomfort and did not require any additional treatment. No severe arrhythmias, i.e. atrial or ventricular fibrillation or flutter were registered. No ischemic heart changes were recorded either (Table 4).

Two patients manifested a significant blood pressure decrease, these were the patients with urticaria/ angioedema and airway obstruction (Table 5). One of the subjects required typical treatment of anaphylaxis, including adrenaline, while the second one, despite a significant reduction in blood pressure, did not feel any discomfort and did not require any additional intervention.

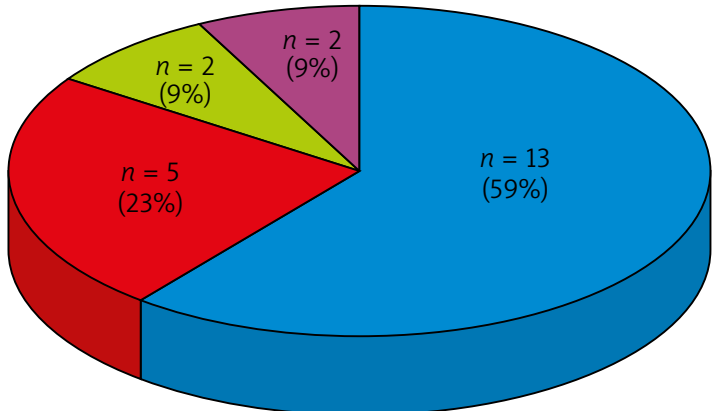

$59 \%(n=13)$ Urticaria and/or angioedema

23\% $(n=5)$ Respiratory response

$\square 9 \%(n=2)$ Urticaria/angioedema and respiratory response

9\% $(n=2)$ Urticaria/angioedema, respiratory response and hypotension

Figure 1. Types of responses in the patients group with positive oral aspirin challenge results

\section{Discussion}

The study concluded that in the group of patients with suspected hypersensitivity to NSAIDs in anamnesis, OAC (+) confirms the suspicion in $\sim 42 \%$ of patients. Detailed monitoring and analysis of the cardiovascular reaction allow to conclude that, although possible, they are not frequent and despite the theoretical premises, with the currently recommended method of qualifying patients for OAC and the methods of its implementation, do not seem to pose any serious health and life risk to the patients. It is noteworthy, however, that the

Table 4. Arrhythmia and conduction disorders during oral aspirin challenge

\begin{tabular}{|c|c|c|c|c|c|c|c|c|c|c|c|c|}
\hline \multirow[t]{3}{*}{ Parameter } & \multicolumn{6}{|c|}{ Day 1 - placebo } & \multicolumn{6}{|c|}{ Day 2 - ASA } \\
\hline & \multicolumn{2}{|c|}{$\begin{array}{l}\text { All subjects } \\
\quad(n=52)\end{array}$} & \multicolumn{2}{|c|}{$\begin{array}{l}\text { OAC }(+) \\
(n=22)\end{array}$} & \multicolumn{2}{|c|}{$\begin{array}{l}\text { OAC }(-) \\
(n=30)\end{array}$} & \multicolumn{2}{|c|}{$\begin{array}{l}\text { All subjects } \\
\quad(n=52)\end{array}$} & \multicolumn{2}{|c|}{$\begin{array}{l}\mathrm{OAC}(+) \\
(n=22)\end{array}$} & \multicolumn{2}{|c|}{$\begin{array}{l}\text { OAC }(-) \\
(n=30)\end{array}$} \\
\hline & $n$ & $\%$ & $n$ & $\%$ & $n$ & $\%$ & $n$ & $\%$ & $n$ & $\%$ & $n$ & $\%$ \\
\hline Tachycardia & 40 & 77 & 17 & 77 & 23 & 77 & 38 & 73 & 19 & 86 & 19 & 63 \\
\hline SVex & 29 & 56 & 11 & 50 & 18 & 60 & 26 & 50 & 10 & 45 & 16 & 53 \\
\hline SVT & 4 & 8 & 0 & 0 & 4 & 13 & 3 & 6 & 1 & 4 & 2 & 7 \\
\hline $\mathrm{AF}$ & 0 & 0 & 0 & 0 & 0 & 0 & 0 & 0 & 0 & 0 & 0 & 0 \\
\hline AFib & 0 & 0 & 0 & 0 & 0 & 0 & 0 & 0 & 0 & 0 & 0 & 0 \\
\hline Vex & 25 & 48 & 12 & 54 & 13 & 43 & 29 & 56 & 11 & 50 & 18 & 60 \\
\hline VT & 1 & 2 & 0 & 0 & 1 & 3 & 1 & 2 & 1 & 4 & 0 & 0 \\
\hline VFI & 0 & 0 & 0 & 0 & 0 & 0 & 0 & 0 & 0 & 0 & 0 & 0 \\
\hline VFib & 0 & 0 & 0 & 0 & 0 & 0 & 0 & 0 & 0 & 0 & 0 & 0 \\
\hline Bradycardia & 26 & 50 & 9 & 40 & 17 & 57 & 24 & 46 & 9 & 40 & 15 & 50 \\
\hline AV block & 2 & 4 & 2 & 9 & 0 & 0 & 1 & 2 & 1 & 4 & 0 & 0 \\
\hline
\end{tabular}

SVex - supraventricular extrasystoles, SVT - supraventricular tachycardia, AF-atrial flutter, AFib - atrial fibrillation, Vex - ventricular extrasystoles, VT - ventricular tachycardia, VF-ventricular flutter, VFib-ventricular fibrillation, AV block - atrioventricular block, OAC(+) - patients with positive oral aspirin challenge results, $O A C(-)$ - patients with a negative oral aspirin challenge results. 
Table 5. Changes in blood pressure during oral aspirin challenge

\begin{tabular}{|c|c|c|c|c|c|c|c|c|c|c|c|c|}
\hline \multirow[t]{3}{*}{$\mathrm{BP}$} & \multicolumn{6}{|c|}{ Day 1 - placebo } & \multicolumn{6}{|c|}{ Day 2 - ASA } \\
\hline & \multicolumn{2}{|c|}{$\begin{array}{c}\text { OAC(-) } \\
(n=30)\end{array}$} & \multicolumn{2}{|c|}{$\begin{array}{c}\text { OAC(+) } \\
(n=22)\end{array}$} & \multicolumn{2}{|c|}{$\begin{array}{l}\text { All subjects } \\
\quad(n=52)\end{array}$} & \multicolumn{2}{|c|}{$\begin{array}{c}\text { OAC(-) } \\
(n=30)\end{array}$} & \multicolumn{2}{|c|}{$\begin{array}{c}\text { OAC(+) } \\
(n=22)\end{array}$} & \multicolumn{2}{|c|}{$\begin{array}{l}\text { All subjects } \\
\quad(n=52)\end{array}$} \\
\hline & $n$ & $\%$ & $n$ & $\%$ & $n$ & $\%$ & $n$ & $\%$ & $n$ & $\%$ & $n$ & $\%$ \\
\hline Reduced & 0 & 0 & 0 & 0 & 0 & 0 & 0 & 0 & 2 & 9 & 2 & 4 \\
\hline Elevated & 4 & 13 & 10 & 45 & 14 & 27 & 3 & 10 & 10 & 45 & 13 & 25 \\
\hline
\end{tabular}

Reduced - systolic BP of less than $90 \mathrm{~mm} \mathrm{Hg}$ and/or rapid BP reduction by $\geq 30 \mathrm{~mm} \mathrm{Hg}$ from the output value. Elevated - defined as the average value of day and night measurements above $130 / 80 \mathrm{~mm} \mathrm{Hg}, O A C(+)$ - patients with a positive oral aspirin challenge, OAC(-) - patients with a negative oral aspirin challenge.

registered reactions did not always have enough clinical significance for the patients to report them, which would act as a signal to halt the test. Practical conclusions from this last statement must also be cautious, because the presented studies involved a relatively small group of 52 subjects. This stemmed from the necessity to adhere to the recommended OAC protocols (mainly the qualification of patients for the study) as well as simultaneous monitoring of the cardiovascular and respiratory systems, which was not always accepted by the patients. It should be noted, however, that almost all previously published analyses concerning similar problems of running OAC involved similar groups of several dozen patients [17, 18]. For obvious reasons (no indications), for the purpose of the present work, no parallel studies have been planned for the healthy, i.e. the implementation of OAC in patients with a negative history of hypersensitivity to NSAIDs. In the course of OAC, isolated urticaria and/or angioedema occurred most frequently. This concerned almost $25 \%$ of patients and $60 \%$ of patients with a positive OAC result. This was consistent with the results of virtually all analyses that addressed the problem of the prevalence of hypersensitivity symptoms to NSAIDs [17]. In the studies by Kasper et al. concerning hypersensitivity to ASA in patients with asthma, as also shown in the present study, the most frequently reported form was urticarial oedema, which was found in $\sim 60 \%$ of patients, and isolated urticaria was observed only in $33.3 \%$ [17].

No studies analysing cardiovascular reactions during OAC were found in the available literature, although, as demonstrated in the introduction, there are theoretical reasons for such reactions. This is mainly due to the currently adopted method of interpretation of the OAC result, which is based on the feedback reported by patients, confirmed later by doctor's examination, using objective tests such as spirometry performed at selected time points and after reporting symptoms justifying their implementation. ECG is not usually performed routinely, but only when the respondents report pain in their chest area, although then the most expected symptoms are related to the respiratory system and such examination is performed occasionally. Hypotension is one of the extreme manifestations of hypersensitivity. During the controlled administration of ASA, the appearance of first symptoms results in discontinuation of the test and implementation of an appropriate procedure. During the course of OAC, a decrease in BP was registered in 2 patients, while only 1 patient required treatment typical for shock.

It should be emphasized that the patients qualified for the study have not been treated for cardiovascular diseases before, i.e. hypertension, arrhythmia or coronary heart disease, and they have not reported these symptoms, but in some of them, during OAC an increase in blood pressure (BP) values was observed both on the day of placebo and ASA administration; in the group with $\mathrm{OAC}(+)$, BP values were significantly higher than in the group with $\mathrm{OAC}(-)$. As expected, in the majority of patients with $\mathrm{OAC}(+)$, the systolic and diastolic mean values were lower than on the placebo day, but these were not statistically significant changes. Similar results were obtained in the group with $\mathrm{OAC}(-)$. It seems that the OAC procedure itself, which assumes the administration of increasing doses of ASA at time intervals, continuous clinical monitoring and early interruption of the test and administering adrenaline, fluids, antihistamines and glucocorticosteroids, depending on the needs, prevented the development of severe hypersensitivity reactions, which could have reduced BP.

Interestingly, there are no cardiac monitoring studies in relation to other provocative tests used in allergology, although it has been known since the 1930s that such a reaction is quite common. The so-called idioblapsis, i.e. a family running IgE-independent response to food, manifested in pulse acceleration was the subject of AF Coca study. In the so-called Coca's test, which is now of historical importance, acceleration of the heart rate by 10-20 beats per minute after consumption of a specific food was supposed to indicate hypersensitivity to its components [19]. Similarly to the food allergy described by Coca, tachycardia was the expected phenomenon during OAC. However, in my research, it was registered in more than $70 \%$ of respondents with no difference to the result of OAC, but both on the day of placebo and ASA administration. At the same time, the mean heart rate in the period of symptoms increased by $\sim 7-11$ beats per minute. However, the literature reports cases of atri- 
al fibrillation in patients with an acute allergic reaction, e.g. after an insect bite [20]. Some authors argue that atrial fibrillation episodes can be triggered by allergic reactions that may be caused by an increase in proinflammatory cytokines such as interleukin 6 (IL-6). Other authors explain this with the pro-arrhythmogenic effect of mediators of allergic reactions, especially the effect of histamine on the heart-conduction system [21]. In the current study, the majority of patients suffered from various types of cardiac dysfunctions (up to $90 \%$ of subjects) most often in the form of tachycardia, followed by single SVEx and VEx. However, this occurred to a similar degree on the day of placebo administration and on the day of ASA administration. Also, there were no significant differences in this respect between patients with a positive or negative OAC. Similar results were obtained by Malerba et al. They analysed 46 patients in whom coronary artery disease was excluded and no pro-arrhythmogenic drugs were used, with indications for bronchial methacholine challenge, monitoring the occurrence of arrhythmias before, during and after this test [22]. The authors, both in the positive and negative test groups found a similar increase in the number of additional supraventricular and ventricular stimuli and, what is interesting, rather a slow-down than an acceleration of cardiac function. During OAC, the patients did not report any other symptoms, in particular any chest pains, nor were there any changes in the registered material that would be an indication for early discontinuation of OAC, in particular regarding changes in the ST-T segment, which confirms that ischemic heart symptoms are a very rare manifestation of hypersensitivity, and monitoring by available methods (Holter ECG, DBPM) does not allow to register them.

\section{Conclusions}

There were no clinically significant cardiac arrhythmias recorded during $O A C$, and the observed changes in the records of BP and ECG monitoring show that OAC performed in accordance with the current guidelines does not pose a high risk to the patient's health and life as a result of cardiovascular reactions. However, there is a risk of severe anaphylactic reaction and/or circulation risk during OAC, especially in patients with asthma and airway obstruction after NSAIDs. The fact that not all objectively occurring respiratory and circulatory disorders during the performance of OAC were experienced and reported by patients, as well as the need to provide appropriate assistance in the case of $\mathrm{OAC}(+)$ and to monitor its efficacy, result in a situation where OAC should be performed in a hospital setting.

\section{Conflict of interest}

The authors declare no conflict of interest.

\section{References}

1. Lafont O. From the willow to aspirin. Rev Hist Pharm (Paris) 2007; 55: 209-16.

2. Hirschberg BC. Mitteilung uber einen Fall von Nebenwirkung des Aspirin. Dtsch Med Wochenschr 1902; 28: 416.

3. Gilbert GB. Unusual idiosyncrasy to aspirin. JAMA 1911; 56: 1262.

4. Chafee FH, Settipane GA. Aspirin intolerance. I. Frequency in an allergic population. J Allergy Clin Immunol 1974; 53: 193-9.

5. Hedman J, Kaprio J, Poussa T, et al. Prevalence of asthma, aspirin intolerance, nasal polyposis and chronic obstructive pulmonary disease in a population- based study. Int J Epidemiol 1999; 28: 717-22.

6. Kupczyk M, Kuna P. Aspirin and other nonsteroidal anti-inflammatory drugs hypersensitivity - mechanisms, diagnostics and treatment. Pneumonol Alergol Pol 2008; 76: 366-77.

7. Melillo G, Balzano G, Bianco S, et al. Oral and inhalation provocation tests for the diagnosis of aspirin-induced asthma. Allergy 2001; 56: 899-911.

8. Kounis NG. Kounis syndrome (allergic angina and allergic myocardial infarction): a natural paradigm? Int J Cardiol 2006; 110: 7-14.

9. Kalsner S, Richards R. Coronary arteries of cardiac patients are hyperreactive and contain stores of amines: a mechanism for coronary spasm. Science 1984; 223: 1435-7.

10. Kounis NG, Kouni SN, Koutsojannis CM. Myocardial infarction after aspirin treatment, and Kounis syndrome. J R Soc Med 2005; 988: 296.

11. Kumar A, Berko NS, Gothwal R, et al. Kounis syndrome secondary to ibuprofen use. Int J Cardiol 2009; 137: 79-80.

12. Jutel M, Akdis M, Akdis C. Histamine, histamine receptors and their role in immune pathology. Clin Exp Allergy 2009; 39: 1786-800.

13. Nakamura T, Itadani H, Hidaka Y. Molecular cloning and characterization of a new human histamine receptor HH4R. Biochem Biophys Res Commun 2000; 279: 615-20.

14. Rojas-Perez-Ezquerra P, Noguerado-Mellado B, MoralesCabeza C, et al. Atrial fibrillation in anaphylaxis. Am J Med 2017; 130: 1114-6.

15. Chen Q, Liu T, Li G. Sustained ventricular tachycardia caused by anaphylactic reaction. Am J Emerg Med 2013; 31: 1625. e3-4.

16. Nizankowska E, Bestynska-Krypel A, Cmiel A, et al. Oral and bronchial provocation tests with aspirin for diagnosis of aspirin-induced asthma. Eur Respir J 2000; 15: 863-9.

17. Kasper L, Sladek K, Bochenek G. The frequency of hypersensitivity to non-steroidal anti-inflammatory drugs (NSAIDs) in the population of adult asthmatics in Poland based on an epidemiological questionnaire. Pneumonol Alergol Pol 2009; 77: 431-9.

18. Kim MS, Cho YJ. Flow cytometry-assisted basophil activation test as a safe diagnostic tool for aspirin/NSAID hypersenstivity. Allergy Asthma Immunol Res 2012; 4: 137-42.

19. Coca AF. Familial nonreaginic food-allergy (idioblapsis) Practical management. Int Arch Allergy Appl Immunol 1950; 1: 173-89.

20. Patel SC, Detjen PF. Atrial fibrillation associated with anaphylaxis during venom and pollen immunotherapy. Ann Allergy Asthma Immunol 2002; 89: 209-11.

21. Wolff AA, Levi R. Histamine and cardiac arrhythmias. Circ Res 1986; 58: 1-16.

22. Malerba M, Radaeli A, Politi A, et al. Cardiac arrhythmia monitoring during bronchial provocation test with methacholine. Chest 2003; 124: 813-8. 